PROCEEDINGS OF THE

AMERICAN MATHEMATICAL SOCIETY

Volume 131, Number 3, Pages 905-909

S 0002-9939(02)06611-X

Article electronically published on July 17, 2002

\title{
ON THE OSCILLATION AND PERIODIC CHARACTER OF A THIRD ORDER RATIONAL DIFFERENCE EQUATION
}

\author{
W. T. PATULA AND H. D. VOULOV \\ (Communicated by Carmen C. Chicone)
}

\begin{abstract}
We prove that every positive solution of the following difference equation:

$$
x_{n}=1+\frac{x_{n-2}}{x_{n-3}}, \quad n=0,1, \ldots,
$$

converges to a period two solution.
\end{abstract}

\section{INTRODUCTION}

The nonlinear difference equation

$$
x_{n}=F\left(x_{n-1}, \ldots, x_{n-k}\right), \quad n=0,1, \ldots,
$$

of order $k>1$ has been receiving increasing attention in the last decade. The general assumption is that $F \in C\left[\mathbb{R}^{k}, R\right]$ and that a set, $\mathcal{S}=\left\{x_{-1}, \ldots, x_{-k}\right\}$, of initial values is given. The basic theory of global behavior of solutions of Eq. (1.1) of order greater than one is still in its infancy. Most of the results about Eq. (1.1), $k>1$, treat the case when $F$ is a rational function. For a second order rational difference equation, see the monograph of Kulenovic and Ladas [6] and the references therein. For rational difference equations of order greater than two, several results have been obtained in [1, 2, 3, 4, 5]. Recently, in [7, G. Ladas conjectured that every positive solution of the third order rational difference equation

$$
x_{n}=1+\frac{x_{n-2}}{x_{n-3}}, \quad n=0,1, \ldots,
$$

converges to a period two solution.

Here we prove this conjecture using a general convergence result established in [3]. We also prove that every nontrivial solution of (1.2) oscillates.

\section{Preliminaries}

For the proof of our main convergence result in Section 4, we will need the following global convergence theorem established in [3] (see also 2]).

Theorem A ([3, Theorem 1.1]). Let $F \in C\left[I^{k}, I\right]$ for some interval I of positive real numbers and for some natural number $k$. Then every positive solution of Eq. (1.1) has a finite limit in I if the following statements are true:

Received by the editors May 28, 2001 and, in revised form, October 22, 2001.

2000 Mathematics Subject Classification. Primary 39A10.

Key words and phrases. Periodic solution, semicycles, oscillation.

(C)2002 American Mathematical Society 
(1) $F\left(z_{1}, \ldots, z_{k}\right)$ is nondecreasing in each of its arguments;

(2) $F\left(z_{1}, \ldots, z_{k}\right)$ is strictly increasing in each of the arguments $z_{i_{1}}, \ldots, z_{i_{e}}$, where $i_{1}, \ldots, i_{e}$ are relatively prime;

(3) $F(c, \ldots, c)=c$ for every $c \in I$.

The proof of this theorem is based on the method of full limiting sequences developed by G. Karakostas [4, and it may be found in [3]. It was applied there to investigate the periodic character of the difference equation

$$
x_{n}=\frac{A_{1}}{x_{n-1}}+\cdots+\frac{A_{k}}{x_{n-k}} .
$$

In this paper, we use Theorem A to study the asymptotic behavior of the solutions of Eq. (1.2).

\section{Oscillation}

Consider Eq. (1.2). Once the positive values $x_{-3}, x_{-2}$, and $x_{-1}$ are specified, the solution of (1.2) is uniquely determined. As in [1 p. 3258], we define the equilibrium or trivial solution, $\bar{x}$, to be the solution of (1.2) obtained by setting $x_{n} \equiv c, \forall n$. For Eq. (1.2),

$$
\bar{x}=2 .
$$

We will prove that any nontrivial solution of (1.2) oscillates around $\bar{x}=2$. That is, there will be a "string" of consecutive values of $x_{n}$ such that $x_{n} \geq 2$. This set of elements will be called a positive semicycle. This will be followed by another "string" of consecutive solution values $x_{n}$ such that $x_{n}<2$. This set of elements will be designated as a negative semicycle. Oscillation means that for any $N$, there exist positive and negative semicycles with elements $x_{n}$ where $n>N$.

We have the following theorem.

Theorem 3.1. Any nontrivial solution of Eq. (1.2) oscillates about the equilibrium solution $\bar{x}=2$. Positive and negative semicycles have length at most 5 . Moreover, examples of positive and negative semicycles of length 5 do exist.

Proof. We will prove oscillation by showing there is an upper bound of 5 for the maximum number of elements in both a positive and a negative semicycle. We consider positive semicycles first and argue various cases.

Suppose $x_{n-5}=2$. Consider $x_{n-4}$. If $x_{n-4}<2$, then $x_{n-4}$ begins a negative semicycle. Suppose $x_{n-4}=2$. Then $x_{n-3}>2$, since otherwise $x_{n}$ is the trivial solution. From (1.2), if $x_{n-5}=x_{n-4}=2$, then $x_{n-2}=2$. However, since $x_{n-2}=2$ and $x_{n-3}>2$, (1.2) implies $x_{n}<2$. Thus $x_{n}$ starts a negative semicycle.

Next, suppose $x_{n-4}>2$ (and $x_{n-5}=2$ ). Then (1.2) implies $x_{n-2}>2$. If $x_{n-3}<x_{n-4}$, then $x_{n-1}<2$ and starts a negative semicycle. Thus, suppose $x_{n-3} \geq x_{n-4}$. Then $x_{n-1} \geq 2$. So we have $x_{n-5}=2, x_{n-3} \geq x_{n-4}>2, x_{n-2}>2$, and $x_{n-1} \geq 2$.

Case 1: $x_{n-2}<x_{n-3}$. Then (1.2) implies $x_{n}<2$.

Case 2: $x_{n-2} \geq x_{n-3}$. This means

$$
\begin{aligned}
x_{n-2} & =1+\frac{x_{n-4}}{x_{n-5}} \geq x_{n-3} \geq x_{n-4} \\
& \Rightarrow 1+\frac{x_{n-4}}{2} \geq x_{n-4} \\
& \Rightarrow x_{n-4} \leq 2, \quad \text { a contradiction. }
\end{aligned}
$$


Hence, if $x_{n-5}=2$, there can be at most 4 additional consecutive elements with solution values $\geq 2$.

Suppose $x_{n-5}>2$. If $x_{n-4}<x_{n-5}$, then $x_{n-2}<2$. Thus, we assume $x_{n-4} \geq$ $x_{n-5}(>2)$. If $x_{n-3}<x_{n-4}$, then $x_{n-1}<2$. So, assume $x_{n-3} \geq x_{n-4}$, which means $x_{n-1} \geq 2$. Then

$$
x_{n}=1+\frac{x_{n-2}}{x_{n-3}} \leq 1+\frac{x_{n-2}}{x_{n-4}}=1+\frac{1}{x_{n-4}}+\frac{1}{x_{n-5}}<1+\frac{1}{2}+\frac{1}{2}=2,
$$

which means $x_{n}$ starts a negative semicycle.

In summary, if $x_{n-5} \geq 2$, there can be at most four consecutive additional solution elements with values $\geq 2$.

Next we consider negative semicycles and begin by assuming $x_{n-5}<2$. If $x_{n-4} \geq x_{n-5}$, then (1.2) implies $x_{n-2} \geq 2$, which marks the beginning of a positive semicycle. Thus, we may suppose $x_{n-4}<x_{n-5}$. In a similar fashion, we may assume $x_{n-3}<x_{n-4}$, which means $x_{n-1}<2$. Then

$$
x_{n}=1+\frac{x_{n-2}}{x_{n-3}}>1+\frac{x_{n-2}}{x_{n-4}}=1+\frac{1}{x_{n-4}}+\frac{1}{x_{n-5}}>1+\frac{1}{2}+\frac{1}{2}=2 .
$$

Accordingly, if $x_{n-5}<2$, there can be at most 4 additional consecutive solution elements with values $<2$.

The previous arguments imply that any nontrivial solution of (1.2) oscillates and that positive and negative semicycles can have length at most 5 . To exhibit a positive semicycle of length 5 , let $x_{n-5}=3, x_{n-4}=4$, and $x_{n-3}=4$. To exhibit a negative semicycle of length 5 , let $x_{n-5}=3 / 2, x_{n-4}=5 / 4$, and $x_{n-3}=9 / 8$. This completes the proof of Theorem 3.1.

\section{Period two solutions}

The main result in this section is the following convergence theorem.

Theorem 4.1. Every positive solution of Eq. (1.2) converges to a period two solution.

Proof. Let $\left\{x_{n}\right\}$ denote a positive solution of Eq. (1.2). By Eq. (1.2), we may write

$$
\frac{x_{n}}{x_{n-2}}=\frac{1}{x_{n-2}}+\frac{1}{x_{n-3}} .
$$

Using various iterates of Eq. (1.2), we obtain

$$
\begin{aligned}
x_{n+2}=1+\frac{x_{n}}{x_{n-1}} & =1+\frac{x_{n}}{x_{n-2}} \cdot \frac{x_{n-2}}{x_{n-1}} \\
& =1+\left(\frac{1}{x_{n-2}}+\frac{1}{x_{n-3}}\right) \frac{x_{n-2}}{x_{n-1}} \\
& =1+\frac{1}{x_{n-1}}+\frac{1}{x_{n-3}\left(x_{n+1}-1\right)} .
\end{aligned}
$$

By substituting $(n-3)$ for $(n+2)$ in the last expression and utilizing Eq. (1.2) again, we obtain for $n \geq 5$ that

$$
x_{n}=1+\frac{x_{n-2}}{x_{n-3}}=1+\frac{x_{n-2}}{1+\frac{1}{x_{n-6}}+\frac{1}{x_{n-8}\left(x_{n-4}-1\right)}} .
$$


Equation (4.1) is now in a form where we can consider $n$ as being either even or odd. Specifically, for $q=0,1$, set

$$
n=2 s+q, \quad y_{s}=x_{2 s+q}-1 .
$$

Then Eq. (4.1) becomes

$$
y_{s}=\frac{1+y_{s-1}}{1+\frac{1}{1+y_{s-3}}+\frac{1}{y_{s-2}\left(y_{s-4}+1\right)}} .
$$

Observe that Eq. (4.3) has the same form as Eq. (1.1) with

$$
F\left(z_{1}, z_{2}, z_{3}, z_{4}\right)=\frac{1+z_{1}}{1+\frac{1}{z_{3}+1}+\frac{1}{z_{2}\left(1+z_{4}\right)}}
$$

and $I=(0, \infty)$. It is clear that $F\left(z_{1}, z_{2}, z_{3}, z_{4}\right)$ is strictly increasing in all its arguments and

$$
F(c, c, c, c)=c .
$$

Therefore by Theorem A, $y_{n}$ converges. Hence, by (4.2), it follows that the sequences $\left\{x_{2 s}\right\}$ and $\left\{x_{2 s+1}\right\}$ are both convergent, which completes the proof.

Corollary 4.2. Equation (1.2) has infinitely many period 2 solutions. More precisely, every positive period two solution of Eq. (1.2) is generated by the initial conditions

$$
x_{-1}=x_{-3}=a, \quad x_{-2}=\frac{a}{a-1}
$$

for some $a>1$.

Proof. It is easy to verify that Eq. (1.2) with the initial conditions (4.4), for any $a>1$, generates a period two solution of Eq. (1.2). On the other hand, let $\left\{x_{n}\right\}$ be a period two solution of Eq. (1.2), i.e.,

$$
x_{2 s}=a, \quad x_{2 s-1}=b \quad \text { for every } s \geq-1 .
$$

Then, by Eq. (1.2), with $n=2 s$, we obtain

$$
a=1+\frac{a}{b}>1 \quad \text { and } \quad b=\frac{a}{a-1},
$$

which completes the proof.

\section{REFERENCES}

[1] R. DeVault, G. Ladas, and S.W. Schultz, On the recursive sequence $x_{n+1}=\frac{A}{x_{n}}+\frac{1}{x_{n-2}}$, PAMS 126 (1998), 3257-3261. MR 99a:39009

[2] H. El-Metwally, E.A. Grove, and G. Ladas, A global convergence with applications to periodic solutions, J. Math. Anal. Appl. 245 (2000), 161-170. MR 2001g:39014

[3] H. El-Metwally, E.A. Grove, G. Ladas, and H.D. Voulov, On the global attractivity and the periodic character of some difference equations, J. Diff. Eqn. Appl. 7 (2001), 837-850.

[4] G. Karakostas, Asymptotic 2-periodic difference equations with diagonally self-intervertebral responses, J. Diff. Eqn. Appl. 6(3) (2000), 329-335. MR 2001d:39004

[5] G. Karakostas, Convergence of a difference equation via the full limiting sequences method, Diff. Eqn. and Dyn. Sys. 1 (1993), 289-294. MR 95c:34019 
[6] M.R.S. Kulenovic and G. Ladas, Dynamics of Second-Order Rational Difference Equations with Open Problems and Conjectures, Chapman and Hall/CRC, 2002.

[7] G. Ladas, Open problems and conjectures, AMS Joint Math. Meetings, January 2001 (New Orleans), Program \#364.

Department of Mathematics, Southern Illinois University Carbondale, Carbondale, ILLINOIS 62901-4408

E-mail address: wpatula@math.siu.edu

Department of Mathematics, Southern Illinois University Carbondale, Carbondale, ILLINOIS 62901-4408

E-mail address: voulovh@yahoo.com 